BIO Web of Conferences 1, 00005 (2011)

DOI: $10.1051 /$ bioconf $/ 20110100005$

(C) Owned by the authors, published by EDP Sciences, 2011

\title{
Building of action and representation of action during infancy, childhood and adolescence.
}

\author{
Christine ASSAIANTE
}

\author{
CNRS- Université De Provence, Equipe Développement et Pathologie de l'Action, Pôle 3C - \\ Laboratoire Neurosciences Intégratives \& Adaptatives (UMR 6149), Centre St Charles - Case B, \\ 3 Place Victor Hugo 13331 Marseille Cedex 03 - France \\ Email: christine.assaiante@univ-provence.fr
}

\begin{abstract}
The early action/perception matching, subserving the motor simulation network, is probably a major milestone for the building of action and representation of action during the course of ontogenesis. We have developed a functional approach of motor development based on a gradual mastering of coordination, adaptation and anticipation in postural control in the course of ontogenesis from babies to adolescents. This functional approach is recently associated with studies of brain structures involved in action and representation of action in children and adolescents. From our developmental studies, it was possible to put in light two turning points during motor development, such as 6/7 years of age and adolescence. The first step for children consists in building a repertoire of postural strategies. The second step consists in learning to select postural strategy depending on the characteristics of the task and the environmental requirements. An appropriate selection means to anticipate the consequence of the movement in order to maintain balance control and efficiency of the task. Taking into account the complexity of the parameters to control and the late maturation of anticipation and representation of action, it is not surprising that the development of postural control continues up to late periods during childhood and adolescence.
\end{abstract}

\section{Introduction}

From birth, one essential aspect of motricity is to achieve a fundamental adaptive function: communication. This is enabled by a constant dialog between child and its environment, in particular through sensori-motor stimulations and, already, at a very early age including spontaneous movement during babyhood. Our developmental studies based on functional approach, are focused 1. on the development of coordination between posture and movement and also between the various body segments involved in action ; 2. on the development of anticipation that compensate in advance for the destabilizing effect of the action on the basis on internal representation ; 3 . on the development of adaptation to the new internal constraints of the subject, to the tasks and the environment. Therefore, postural development is totally integrated to the building of cognitive motor development that implies permanent adaptations to various contexts and environmental demands, including also social interactions.

An important function of posture is to ensure maintenance of balance during the initiation, continuance and completion of action. In addition, posture serves as a reference frame for the production of accurate movements. Indeed, the efficient action of the body in space and its representation need that the central nervous system (CNS) uses a reference frame around which the external objects' positions and displacements could be estimated and movements can be built up. A question asked frequently in motor neuroscience surrounds the problem of the existence of a stable reference frame used to control posture and voluntary movement $[1,2]$.

\section{Building a repertoire of balance strategies during infancy and childhood}

Studies on the development of balance control have reported the existence of marked differences with respect to adults, especially in terms of the segmental stabilization occurring at head, trunk and pelvis levels $[3,4,5]$. According to their ontogenetic model of balance control, Assaiante and Amblard [3] assumed that the various balance strategies adopted by children as well as by adults involve to take into account two

This is an Open Access article distributed under the terms of the Creative Commons Attribution-Noncommercial License 3.0, which permits unrestricted use, distribution, and reproduction in any noncommercial medium, provided the original work is properly cited. 
main functional principles of spatial organization. The first concerns the choice of the stable reference frame on which the equilibrium control is based, and the second concerns the gradual mastery of the degrees of freedom of the various body joints. The choice of the stabilized anatomical segment of reference as well as the character of coupling between articulations depends on the dynamic constraints determining the difficulty of a motor task, the environment and the characteristics of each developmental period. For example, the pelvis constitutes the first stable reference frame, around which balance control can be built up, as soon as independent locomotion is acquired [5, 6]. By contrast, controlling head stabilization during locomotor activities constitutes a complex motor skill that takes a long time to mature during childhood [4]. Moreover, the development of head control is not linear. In fact, the development of the head stabilization in space displays a transition phase between 6 and 7 years of age. The beginning of systematic use of the head stabilization in space strategy in 7-year-old children walking on narrow supports was preceded in 6-year-old children by a sort of regression to adopt the alternative head stabilization on trunk strategy. These results particularly emphazise the turning point between 6 et 7 years of age in the development of balance strategies [4, 3].

\section{Development of anticipation during infancy and childhood}

In the past, we proposed some studies aimed at studying the development of the function of anticipation that enables the world to be predictable and coherent. Exploring some motor markers and their cortical correlates in lifting tasks, we found that the function of anticipation needs time to mature during childhood and probably reflects the slow building of action representations $[7,8,9,10]$. The body scheme, slowly built during childhood by integration between vestibular, visual and somatosensory information, contributes to the development of the internal representation of action that constitutes the base of feed-forward control to compensate in advance for the destabilizing effects of the movement.

It has been demonstrated that body stability largely depends on the capacity of the CNS to anticipate the destabilising events. It is not known when in the course of human ontogenesis postural anticipation emerges at the trunk level and whether it is efficient before the acquisition of upright locomotion. Haas et al. [11] have reported lack of anticipation as the principal cause of postural instability in children under 2 years of age. Assaiante et al [6] have observed postural preparation preceding step initiation as soon as 1-4 months after the acquisition of walking. However, anticipatory postural adjustments (APA) did not appear consistently until 4-5 years of age [12]. The APA become more constant only at about 4 years of age but their temporal characteristics are not yet those of adults $[13,11]$.

In order to determine the earliest age of emergence of APA and their efficiency in trunk stabilization, we exposed subjects at three developmental stages to continuous rocking movements of a seat. Choosing a sitting position for all subjects allowed including prewalking children (as young as 6 months) for a direct comparison with young walkers and adults. The crosscorrelation analysis of the EMG signals and the angular displacement of the seat revealed major differences in the timing of the muscle contractions with respect to the movement of the seat between the three tested groups. The best postural performance of the adult group was assured by rhythmic lumbar muscle contractions that peaked before the turning points of the seat. This postural anticipation was absent in the pre-walker group, who showed the largest trunk excursions, but it existed in children who could stand and in early walkers where it corresponded to better trunk stabilisation. The major finding that emerges from this study is that amelioration of dynamic stability is closely related to anticipation and that this ability is rudimentary in non-walking children compared to early walkers. We argue that the ability to anticipate perturbations may be such a rate-limiting factor in transition from sitting to independent walking.

\section{Adaptation of postural reference frames during childhood}

When studying the emergence of postural strategies, it is essential to distinguish between results due to biomechanical reasons strictly and those reflecting the maturation of the CNS. To address this problem, we have studied various types of postural adaptation based either on specific constraints given by the character of the task which covered situations of various balance difficulty; or transitory biomechanical constraints, such as using a splint blocking one knee to destabilize the pelvis (AIS) [5].

3- to 4-year-old children are able to stabilize pelvis and shoulders in space when walking on flat ground. Increasing the task difficulty by walking on a narrow line leads to a loss of shoulder stabilization, which can, however, be re-established if the pelvis is destabilized. Early head stabilization in space, as a substitution for the destabilized pelvis that constitutes the first reference frame, does not occur, contrary to our 
original hypothesis. Thus, short-term adaptation strategies are selected strictly from the repertoire already present at the studied age. The corresponding temporal organization is ascending from the stabilized pelvis to the head. If the pelvis is destabilized, the shoulders tend to become the origin of the temporal sequence.

When walking on a flat ground or a beam, 5- to 6year-old children show the same stabilization patterns as the younger group does. On the other hand, with a destabilized pelvis, no systematic shoulder stabilization in space is present, and the head has not yet acquired this ability either. The results confirm that at around the age of 6 , a turning point appears in the development of equilibrium control, as already reported [4, 3]. The corresponding temporal organization is rather shoulder-centered.

Finally, 7- to 8-year-old children stabilize the pelvis, the shoulders, and the head, suggesting an independent control of each anatomical segment while walking on a flat surface. When balance difficulty increases, for example during walking on a narrow surface, the shoulder stabilization disappears. In the most difficult situation with the imposed pelvis destabilization, the head remains stabilized in space, as do the shoulders sometimes. In contrast, with destabilized pelvis, where one would expect a descending mode of temporal organization, the temporal pattern is completely erroneous. In fact, we observed a sort of regression in the temporal organization of balance control that just precedes the establishment of the descending temporal organization from the head stabilized to the feet.

The latter results of the 7- to 8-year group remind us that even though segmental stabilization and temporal organization usually go hand in hand, in developmental periods it is important to emphasize the time-lag between the mastery of the segmental stabilization that occurs first and the mastery of the corresponding temporal organization that occurs in a second time. Various developmental studies in posture [14, 15, 7, 8] also reported that during childhood the control of timing seems to be the most difficult parameter to acquire and seems to be one of the key factors that reflects the maturation of CNS.

\section{Adolescence : a turning point in brain and body}

During ontogenesis, periods of relatively stable increase in body size alternate with periods of accelerated growth. Adolescence is a period of physiological and psychological transition between childhood and adulthood, which is known to involve considerable morphological, structural and functional changes and also a late cortical maturation [16, 17]. During adolescence the body undergoes many physical changes that necessitate an updating of internal models of action. The refinement of internal models may be supported by the maturation of parietal cortex during adolescence [18].

We adopted the working hypothesis that the body scheme disturbances occurring during adolescence may lead subjects to transiently neglect the information provided by the proprioceptive pathway, which is undergoing considerable changes, and that they may rely more strongly on other sensory systems such as vision to control their orientation and stabilize their body. This hypothesis was therefore tested in a recent study of our group [19], by applying slow oscillations below the detection threshold of the semicircular canal system. Indeed, the low frequency oscillations will not be detected by the vestibular system and therefore postural control in the condition of closed eyes has to rely to a large extent on proprioceptive information.

As we previously speculate, the performances of adolescents are much less efficient than those of adults. Moreover, vision plays a predominant role in adolescents' control of orientation and body stabilization, as previously reported to be the case in infants during the period of acquisition of the main motor abilities [3]. This suggests that the mechanisms underlying postural control are still maturing during adolescence that may constitute a transient period of proprioceptive neglect in sensory integration of postural control.

Better understanding adolescence may have a clinical significance for many developmental pathologies because we speculate that postural development is not linear and that adolescence may be a specific phase in the development of postural control. Idiopathic scoliosis (AIS) is a developmental pathology which expresses spinal deformity mainly during adolescence. The question arises as to what effects these huge body changes including spinal deformity may have on AIS' body scheme, which serves as a reference frame to control posture and its vertical representation, action and representation of action. Herman et al. [20] reported that idiopathic scoliosis patients exhibit perceptual impairments, deficits in sensorimotor adaptation, and balance control. In a recent study [21] it was reported that AIS show substantial similarities with CA (control adolescents) in postural orientation control and in balance control. Visual cues improve postural control in CA and AIS. By contrast with our original speculation, AIS, despite their suspected vestibular deficits, transitory neglect proprioceptive information to control their posture in response to very slow 
oscillations of the support, suggesting that developmental effect is dominant with respect to pathologic effect in adolescence. We concluded that static proprioceptive system, as assessed from our protocol, was not affected by the idiopathic scoliosis, while the dynamic proprioceptive system would be affected.

\section{Conclusion}

All the results presented in this paper support the concept of multiple reference frames depending upon the age and upon the constraints of the task. The first step for children consists in building a repertoire of postural strategies. The second step consists in learning to select the most appropriate postural strategy depending on the ability to anticipate the consequence of the action in order to maintain balance control and efficiency of the task. Anticipatory control, despite its early emergence, slowly matures during childhood and adolescence. Moreover, sensorimotor representations and body scheme slowly enrich motor repertoire during ontogenesis. Taking into account the complexity of the building of motor repertoire, it is not surprising that the development of postural control continues up to late periods during childhood and adolescence.

\section{References}

[1] Paillard J. De l'espace corporel a' l'espace écologique. Symposium de l'association de psychologie scientifique de langue franc,aise. Bruxelles, Paris: Presses Universitaires de France. pp 7-54, 1974.

[2] Berthoz A. Reference frames for the perception and control of movement. In: Paillard J, ed. Brain and Space. pp 81-111, 1991.

[3] Assaiante C, Amblard B. An ontogenetic model for the sensori motor organization of balance control in humans. Human Movement Science 14: 13-43, 1995.

[4] Assaiante C, Amblard B. Ontogenesis of head stabilization in space during locomotion in children: influence of visual cues. Experimental Brain Research 93: 499-515, 1993.

[5] Assaiante C, Mallau S, Viel S, Jover M, Schmitz C. Development of postural control during childhood. Neural Plasticity, Volume 12, Nu 1-2. pp 33-42, 2005.

[6] Assaiante, C., Thomachot, B., Aurenty, R. and Amblard, B. Organization of lateral balance control in toddlers during the first year of autonomous walking. Journal of Motor Behavior, 30(2): 114-129, 1998.

[7] Schmitz, C., Martin, N. and Assaiante, C. Development of anticipatory postural adjustments in a bimanual loadlifting task in children. Experimental Brain Research, 126(2):200-4, 1999.
[8] Schmitz, C., Martin, N. and Assaiante, C. Building Anticipatory Postural Adjustments during childhood: Kinematic and Electromyographic data in a bimanual load-lifting task. Experimental brain research, 142: 354364, 2002.

[9] Schmitz, C., Martineau, J., Barthélémy, C., Assaiante, C. Motor Control and Autism: Deficit of Anticipatory Function. Neuroscience Letters, 348; 17-20, 2003.

[10] Martineau, J., Schmitz, C., Assaiante, C., Blanc, R., Barthélémy, C. Event-related desynchronisation during a bimanual load-lifting task in children with autistic disorder. Neuroscience Letters 367, 298-303, 2004.

[11] Haas G; Diener HC, Rapp H, Dichgans J. Development of feedback and feedforward control of upright stance. Dev Med Child Neurol 31: 481-488, 1989.

[12] Assaiante, C., Woollacott, M., Amblard, B. The development of postural anticipatory adjustments during initiation of gait: Kinematic and EMG Analysis. Journal of Motor Behavior. Vol 32, No 3, 211-226, 2000.

[13] Shumway-Cook A, Woollacott MH. The growth of stability: postural control from a developmental perspective. Journal of Motor Behavior 17: 131-147, 1985.

[14] Eliasson A-C, Forssberg H, Ikuta K, Apel I, Westling G, Johansson RS. Development of human precision grip. V. Anticipatory and triggered grip actions during sudden loading. Exp Brain Res 106: 425-33, 1995.

[15] Konczak J, Dichgans J. The development toward stereotypic arm kinematics during reaching in the first 3 years of life. Exp Brain Res 117: 346-354, 1997.

[16] Paus T. Mapping brain maturation and cognitive development during adolescence. Trends in Cognitive Sciences, 9: 60-68, 2005.

[17] Blakemore S, Choudhury S. Development of the adolescent brain : Implications for executive function and social cognition. Journal of Child Psychology and Psychiatry, 47: 296-312, 2006.

[18] Choudhury S, Charman T, Bird V, Blakemore SJ. Development of action representation during adolescence. Neuropsychologia, 45: 255-262, 2007.

[19] Viel, S., Vaugoyeau, M., Assaiante, C. Postural adaptation of the spatial reference frames to microgravity : back to the egocentric reference frame, PlosOne, 5 (4):e10259, 2010.

[20] Herman R, Mixon J, Fisher A, Maulucci R, and Stuyck J. Idiopathic scoliosis and the central nervous system: a motor control problem. The Harrington lecture, 1983. Scoliosis Research Society. Spine (Phila Pa 1976.),10:114, 1985.

[21] Assaiante, C., Mallau, S., Jouve, J.L., Bollini, G., Vaugoyeau, M. Do adolescent idiopathic scoliosis neglect proprioceptive information in sensory integration of postural control? Submitted. 